\title{
Behavioral Modeling of Transmission Line Channels via Linear Transformations
}

\author{
Albert Vareljian \\ albertv@ieee.org \\ Member, IEEE, Canada
}

\begin{abstract}
An approach based on the linear transformation of network port variables is augmented and applied to behavioral modeling of inhomogeneous transmission line channels with lumped and distributed discontinuities. Generic tools Spice-like and Simulink can be used for channel simulation in the frequency or time domain.
\end{abstract}

\section{Introduction}

Recent developments in very high bit rate wireline data transmission applications as 1000BASE-T Gigabit Ethernet (GE) [1], VDSL [2] and other, have motivated continued researcher interest in accurate simulation of data channels based on the unshielded twisted pair (UTP) medium. As the signaling bandwidths approach the order of $100 \mathrm{MHz}$, even relatively minor channel discontinuities such as short jumpers, connectors etc. need to be accounted for in the system design. Such discontinuities cause multiple reflections in the channel, becoming an impairment for the system performance.

An approach based on the linear transformation of network port variables, originally introduced for synthesis of active filters [3], has been augmented and applied to the behavioral modeling of inhomogeneous transmission line channels with lumped and distributed discontinuities. First, a channel with discontinuities is split into a cascade connection of uniform blocks: two-ports, representing lumped (discrete) circuit elements, or homogeneous sections of transmission line. The bidirectional channel model structure is then derived in a regular procedure, using selectively chosen port variable transformation matrices. The overall model consists of a cascade interconnection of the matrix transfer function modules of the two types. Modules of the first type correspond on an element-by-element basis to the channel discrete instances, or homogeneous transmission line sections, whereas the second type are the so called "port-matching" modules. The later serve as an interface between input and output ports for the adjacent modules of the first type. It is shown that the port-matching requirements result from the discontinuity boundaries in the physical channel and yield a matrix transfer function whose elements are in essence the reflection and refraction coefficients.

The overall channel model, referred to as the linear transformation equivalent (LTE), can be readily simulated in time or frequency domain using generic tools like Matlab/Sim- ulink or Spice. Physical phenomena in transmission lines such as skin effect, dielectric loss and characteristic impedance variations can also be modeled as shown.

Homogeneous transmission line sections are described in terms of their corresponding characteristic impedance, propagation delay and rational $s$-domain transfer function approximating insertion loss frequency response. The near and far end echo and transmission waveforms are generated simultaneously.

The proposed method offers certain advantages compared to the approach traditionally used in the communications community based on the frequency domain computation of the channel chain matrix $A B C D$ parameters [4], [5], with subsequent frequency-to-time discrete-domain Laplace inversion by IFFT. The LTE model naturally yields a continuous-time system which can be simulated using analog variable-step tools. This provides means for a relatively straightforward and efficient behavioral simulation and analysis of complex mixed-signal systems, especially under conditions of timing reference frequency drift and/or offset, as is the case, for example, with a pair of data communications transceivers with timing recovery. The method also allows effective what-if, or component variation analysis. Application example is given whereby GE timing recovery test channel is modeled together with a typical transformer line interface.

\section{Linear Transformation Approach}

The linear transformation method was originally developed by Dimopoulos and Constantinides [3] with the main aim of active filter synthesis from the doubly terminated lossless $L C$ ladder filters. The philosophy of the approach is that physical variables (currents and voltages) of a network are first linearly transformed into a new domain where the corresponding relationships are then realized by means of transfer functions. By varying the linear transformation parameters different structures corresponding to the same prototype network may be obtained, possessing features desirable for a targeted realization. Due to the universal nature of the underlaying concept, the basic approach could be adopted for applications in system modelling, as demonstrated in this paper for the case of a transmission line channel with distributed and lumped discontinuities. The concept of linear transformations is briefly presented below. 
(a)

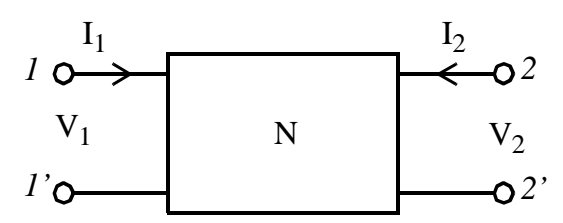

(b)

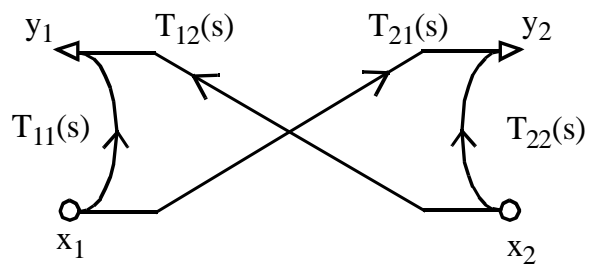

(c)

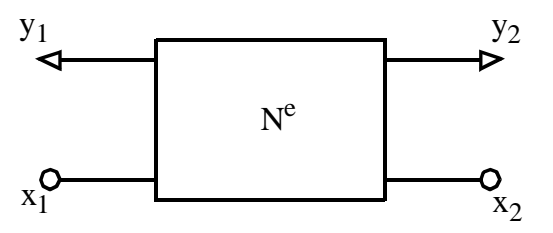

Fig. 1. (a) The passive two-port prototype. (b) The SFG of the corresponding LTE. (c) LTE block diagram.

For an arbitrary linear passive network (see Fig 1(a)) contained between the ports $1-1^{\prime}$ and $2-2^{\prime}$ with associated voltage-current variable vectors $\left[\begin{array}{ll}V_{1} & I_{1}\end{array}\right]^{T}$ and $\left[\begin{array}{ll}V_{2} & I_{2}\end{array}\right]^{T}$, one can assign a two-port $N$ characterized in terms of the modified $^{1}$ chain matrix $A B C D$ as follows:

$$
\left[\begin{array}{c}
V_{1} \\
I_{1}
\end{array}\right]=\left[\begin{array}{ll}
A & B \\
C & D
\end{array}\right]\left[\begin{array}{l}
V_{2} \\
I_{2}
\end{array}\right]=T\left[\begin{array}{l}
V_{2} \\
I_{2}
\end{array}\right] .
$$

The port variable vectors $\left[\begin{array}{ll}V_{1} & I_{1}\end{array}\right]^{T}$ and $\left[\begin{array}{ll}V_{2} & I_{2}\end{array}\right]^{T}$ are linearly transformed by a pair of nonsingular matrices $\Lambda_{1}$ and $\Lambda_{2}$ into the new variables $\left[\begin{array}{ll}x_{1} & y_{1}\end{array}\right]^{T}$ and $\left[\begin{array}{ll}x_{2} & y_{2}\end{array}\right]^{T}$ such that:

$$
\begin{aligned}
& {\left[\begin{array}{l}
x_{1} \\
y_{1}
\end{array}\right]=\left[\begin{array}{ll}
\alpha_{1} & \beta_{1} \\
\gamma_{1} & \delta_{1}
\end{array}\right]\left[\begin{array}{l}
V_{1} \\
I_{1}
\end{array}\right]=\Lambda_{1}\left[\begin{array}{l}
V_{1} \\
I_{1}
\end{array}\right]} \\
& {\left[\begin{array}{l}
x_{2} \\
y_{2}
\end{array}\right]=\left[\begin{array}{ll}
\alpha_{2} & \beta_{2} \\
\gamma_{2} & \delta_{2}
\end{array}\right]\left[\begin{array}{l}
V_{2} \\
I_{2}
\end{array}\right]=\Lambda_{2}\left[\begin{array}{l}
V_{2} \\
I_{2}
\end{array}\right] .}
\end{aligned}
$$

where $\operatorname{det} \Lambda_{1,2}=\Delta_{1,2} \neq 0$.

1. Due to the choice for the port $2-2^{\prime}$ current direction flowing into the network, quantities $B$ and $D$ appear in the modified form with a negative sign in contrast to the conventional notation. (a)

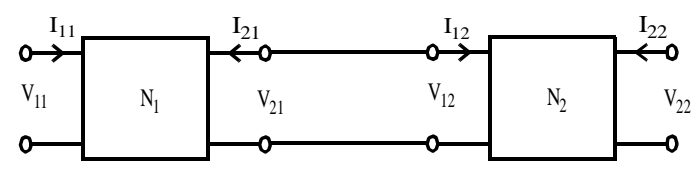

(b)

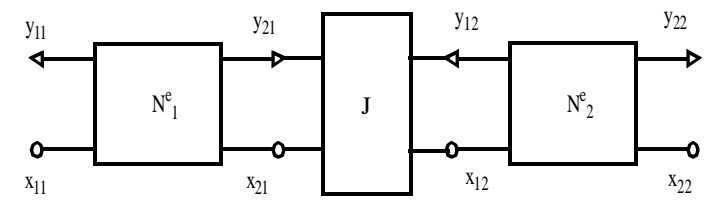

Fig. 2. (a) The cascade connection of two-ports. (b) The interconnection of the corresponding LTE blocks.

Combining (1) and (2) yields:

$$
\left[\begin{array}{l}
x_{1} \\
y_{1}
\end{array}\right]=\Lambda_{1} T \Lambda_{2}^{-1}\left[\begin{array}{l}
x_{2} \\
y_{2}
\end{array}\right]=\frac{1}{\Delta_{2}}\left[\begin{array}{ll}
a & -b \\
c & -d
\end{array}\right]\left[\begin{array}{l}
x_{2} \\
y_{2}
\end{array}\right]
$$

where

$$
\begin{aligned}
& a=\alpha_{1}\left(\delta_{2} A-\gamma_{2} B\right)+\beta_{1}\left(\delta_{2} C-\gamma_{2} D\right) \\
& b=\alpha_{1}\left(\beta_{2} A-\alpha_{2} B\right)+\beta_{1}\left(\beta_{2} C-\alpha_{2} D\right) \\
& c=\gamma_{1}\left(\delta_{2} A-\gamma_{2} B\right)+\delta_{1}\left(\delta_{2} C-\gamma_{2} D\right) \\
& d=\gamma_{1}\left(\beta_{2} A-\alpha_{2} B\right)+\delta_{1}\left(\beta_{2} C-\alpha_{2} D\right)
\end{aligned}
$$

As the chain matrix generally represents inverse (noncausal) input-output relations, it is desirable to group the system variables such that a causal and, therefore, realizable relationships result. If, for example, vector $\left[\begin{array}{ll}y_{1} & y_{2}\end{array}\right]^{T}$ is expressed in terms of $\left[\begin{array}{ll}x_{1} & x_{2}\end{array}\right]^{T}$, the corresponding (realizable) relationship can be found from (3) for any passive $N$, assuming the basic passive network theory identity $\operatorname{det} T=-1^{2}$ as follows:

$$
\left[\begin{array}{l}
y_{1} \\
y_{2}
\end{array}\right]=\left[\begin{array}{cc}
d / b & -\Delta_{1} / b \\
-\Delta_{2} / b & a / b
\end{array}\right]\left[\begin{array}{l}
x_{1} \\
x_{2}
\end{array}\right]=\left[\begin{array}{cc}
T_{11}(s) & T_{12}(s) \\
T_{21}(s) & T_{22}(s)
\end{array}\right]\left[\begin{array}{l}
x_{1} \\
x_{2}
\end{array}\right] .
$$

Equation (4) represents a matrix transfer function whose SFG is shown in Fig. 1(b). A two-input-two-output nonreciprocal (directional) network $N^{e}$ shown in Fig. 1(c) will in general be required for the matrix transfer function realization in (4).

2. The minus sign appears as a result of the chosen direction for $I_{2}$. 
For the purposes of this work the SFG structure of Fig. 1(b) and the realizing network $N^{e}$ of Fig. 1(c) are referred to as the linear transformation equivalent (LTE) of the corresponding passive network $N$. The directional nature of the LTE is reflected in Fig. 1(c) whereby the signals $y_{1}$ and $y_{2}$ are the designated outputs (denoted by triangles) generated in response to the excitations $x_{1}$ and $x_{2}$ (denoted by small circles) that are enforced at the inputs.

Cascade connection of the two-ports $N_{1}, N_{2}$ shown in Fig. 2(a) described in the $V-I$ domain by

$$
\left[\begin{array}{l}
V_{12} \\
I_{12}
\end{array}\right]=\left[\begin{array}{rr}
1 & 0 \\
0 & -1
\end{array}\right]\left[\begin{array}{l}
V_{21} \\
I_{21}
\end{array}\right]
$$

yields together with (1) for the corresponding vector variables of the adjacent LTEs the following relationship:

$$
\left[\begin{array}{l}
x_{12} \\
x_{21}
\end{array}\right]=\left[\begin{array}{cc}
\frac{\alpha_{12} \delta_{21}+\gamma_{21} \beta_{12}}{\gamma_{12} \delta_{21}+\gamma_{21} \delta_{12}} & \frac{\Delta_{12}}{\gamma_{12} \delta_{21}+\gamma_{21} \delta_{12}} \\
\frac{\Delta_{21}}{\gamma_{12} \delta_{21}+\gamma_{21} \delta_{12}} & \frac{\alpha_{21} \delta_{12}+\gamma_{12} \beta_{21}}{\gamma_{12} \delta_{21}+\gamma_{21} \delta_{12}}
\end{array}\right]\left[\begin{array}{l}
y_{12} \\
y_{21}
\end{array}\right]
$$

where the second subscript denotes the index of the respective block. The interconnecting network $J$ in Fig. 2(b) is defined for the adjacent LTEs as a block, realizing the matrix transfer function in (6):

$$
\left[\begin{array}{l}
x_{12} \\
x_{21}
\end{array}\right]=\left[\begin{array}{ll}
J_{11} & J_{12} \\
J_{21} & J_{22}
\end{array}\right]\left[\begin{array}{l}
y_{12} \\
y_{21}
\end{array}\right] .
$$

The later combines signals $y_{12}$ and $y_{21}$ to generate $x_{12}$ and $x_{21}$.

It can be observed from the above considerations that by varying the transformation matrices in (2), different LTE representations in (4) can be obtained for a given prototype. Throughout the rest of this paper transformation matrices of the form

$$
\Lambda_{1}=\Lambda_{2}=\left[\begin{array}{rr}
1 & \beta \\
1 & -\beta
\end{array}\right]
$$

will be employed, resulting in the wave-flow [6], representation in the LTE. With the above assumption, variables $x$ and $y$ have a meaning of incident and reflected voltage waves respectively. Using (7) and assuming only symmetrical networks for $N(A=-D)$, the LTE transfer functions in (4) can be expressed as follows:

$$
\begin{aligned}
& T_{11}=T_{22}=\frac{-(B / \beta+\beta C)}{2 A-B / \beta+\beta C} \\
& T_{12}=T_{21}=\frac{2}{2 A-B / \beta+\beta C}
\end{aligned}
$$

With (7) the matrix transfer function (6) will be simplified:

$$
\left[\begin{array}{l}
x_{12} \\
x_{21}
\end{array}\right]=\left[\begin{array}{ll}
\frac{\beta_{1}-\beta_{2}}{\beta_{1}+\beta_{2}} & \frac{2 \beta_{2}}{\beta_{1}+\beta_{2}} \\
\frac{2 \beta_{1}}{\beta_{1}+\beta_{2}} & \frac{\beta_{2}-\beta_{1}}{\beta_{1}+\beta_{2}}
\end{array}\right]\left[\begin{array}{l}
y_{12} \\
y_{21}
\end{array}\right]
$$

where the single subscript corresponds to the network index.

\section{Homogeneous Transmission Line Sections}

Homogeneous section of a transmission line can be described as a two-port with the modified chain matrix of the form

$$
T=\left[\begin{array}{cc}
\cosh \theta l & -Z_{0} \sinh \theta l \\
\frac{1}{Z_{0}} \sinh \theta l & -\cosh \theta l
\end{array}\right]
$$

where $l$ is the length of the section, $\theta=\sqrt{(R+s L)(G+s C)}$ and $Z_{0}=\sqrt{(R+s L) /(G+s C)}$ are the propagation constant and characteristic impedance of the line with primary constants $R, L, G, C$ per unit length. In practice, the line primary "constants" are actually frequency dependent due to the skin effect in conductors and dielectric loss in the insulator material. To reflect this fact we adopt the following approximations [7], [8] for the series resistance $R$ and shunt conductance $G$ :

$$
\begin{aligned}
R & =r+k \sqrt{s} \\
G & =g+g_{d} Y_{d}(s)
\end{aligned}
$$

where $r, g$ are the $d c$ resistance and leakage respectively, $k$ is the skin effect constant, $g_{d}$ is a constant, and

$$
Y_{d}(s)=\frac{s}{s+\omega_{d}}
$$

represents the dielectric loss frequency response with a pole at angular frequency $\omega_{d}$.

To model a section of homogeneous transmission line we choose in (7) $\beta=Z_{0}$. Thus, from (8) and (10) we can find for the corresponding LTE: $T_{11}=T_{22}=0$, and

$$
T_{12}=T_{21}=e^{-\theta l} .
$$

The interpretation of the above transfer equations is that there are no reflections within the line section, and the line transfer function is of the same form when terminated at both ends by its characteristic impedance $Z_{0}$. When two line sections with different characteristics $\beta_{1}=Z_{01}$ and $\beta_{2}=Z_{02}$ are connected, the LTE interconnection relationship can be found form (9): 


$$
\left[\begin{array}{l}
x_{12} \\
x_{21}
\end{array}\right]=\left[\begin{array}{ll}
\frac{Z_{01}-Z_{02}}{Z_{01}+Z_{02}} & \frac{2 Z_{02}}{Z_{01}+Z_{02}} \\
\frac{2 Z_{01}}{Z_{01}+Z_{02}} & \frac{Z_{02}-Z_{01}}{Z_{01}+Z_{02}}
\end{array}\right]\left[\begin{array}{l}
y_{12} \\
y_{21}
\end{array}\right] .
$$

The elements of the above matrix can be readily recognized as reflection (on the diagonal) and refraction coefficients at the discontinuity boundary for the left and right going voltage waves. With this, the LTE interconnection network function has a meaning of a port-matching block. Naturally, for the adjacent line sections with identical characteristic impedance $Z_{01}=Z_{02}$ the port-matching block is not needed (no discontinuity is present), so $x_{12}$ can be directly connected to $y_{21}$ and $x_{21}$ to $y_{12}$ in a cross-connect fashion shown in Fig. 3. This follows from (15), which with $Z_{01}=Z_{02}$ becomes:

$$
\left[\begin{array}{l}
x_{12} \\
x_{21}
\end{array}\right]=\left[\begin{array}{ll}
0 & 1 \\
1 & 0
\end{array}\right]\left[\begin{array}{l}
y_{12} \\
y_{21}
\end{array}\right] \text {. }
$$

We will now consider transmission line transfer function approximation in (14). The solution to the approximation problem is sought in the form of a cascade of two transfer functions:

$$
e^{-\theta l}=e^{-s \tau_{d}} \cdot e^{-\theta_{1} l}=e^{-s \tau_{d}} \cdot H(s)
$$

where the exponential term on RHS of (17) represents the wave front propagation delay $\tau_{d}=l \sqrt{L C},(s \rightarrow \infty)$, and $H(s)$ is a rational minimal-phase function, approximating the line insertion loss frequency response $\exp \left(-\theta_{1} l\right)$. For sufficiently high frequencies (above $100-200 \mathrm{kHz}$ ) $\theta_{1}$ can be expressed as

$$
\theta_{1} \approx 0.5\left[g_{d} R_{0} Y_{d}(s)+\frac{k}{R_{0}} \sqrt{s}+\frac{r}{R_{0}}\right]
$$

where

$$
R_{0}=\lim _{s \rightarrow \infty} Z_{0}=\sqrt{L / C}
$$

is a purely resistive value observed for the line characteristic impedance at high frequencies. This assumption is well justified for the frequency bands utilized in very high bit rate applications.

For a homogeneous section of transmission line with a given length $l$ the insertion loss frequency response can be computed using (18) and fitted in the frequency domain by a rational function

$$
H(s)=\frac{b_{m} s^{m}+b_{m-1} s^{m-1}+\ldots+b_{1} s+b_{0}}{s^{n}+a_{n-1} s^{n-1}+\ldots+a_{1} s+a_{0}}
$$

where $m \leq n$.

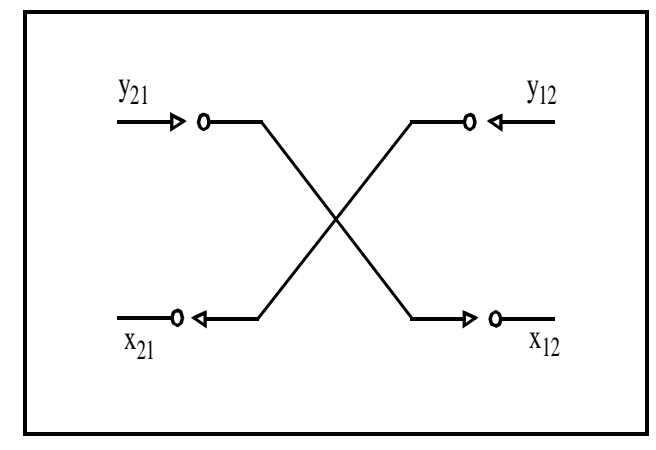

Fig. 3. The LTE cross-connect method.

The frequency fit can be performed with the help of Matlab Signal Processing Toolbox function "invfreqs" [9]. Results of such approximation are shown in Fig. 4 for the case of measured response of a $100 \mathrm{~m}$ category 5 (CAT5) UTP cable used in GE. The following line electric parameters: $r=93.8 \mathrm{~m} \Omega$ / $\mathrm{m}, L=0.613 \mu \mathrm{H} / \mathrm{m}, C=56 \mathrm{pF} / \mathrm{m}, g=0.1 \mathrm{pS} / \mathrm{m}, g_{d}=0.1 \mathrm{mS} /$ $\mathrm{m}, \omega_{d}=2 \pi 90 \mathrm{e} 6 \mathrm{rad} / \mathrm{s}$, and $k=0.29 \mathrm{~m} \Omega / \mathrm{m} / \mathrm{s}^{-1 / 2}$ for the skin effect constant have been chosen such that a good match was observed for the measured and computed based on (18) responses. Fitting was then performed with $m=4$ and $n=6$ for the computed data with frequency points normalized to $125 \mathrm{MHz}^{1}$ to improve accuracy. Corresponding normalized polynomial coefficients for $H(\mathrm{~s})$ in (20) are given in Table I. The UTP cable characteristic shown in Fig. 4 was captured with $100 \Omega$ terminations at both ends. As it can be seen from the graph, the approximation matches very well with the measured data.

TABLE I

NORMALIZED COEFFICIENTS FOR CAT5 (100m) CABLE RESPONSE APPROXIMATION $H(s)$

\begin{tabular}{ccc}
\hline$i$ & $a_{i}$ & $b_{i}$ \\
\hline 0 & 65.412 & 65.139 \\
1 & 1360.2 & 767.03 \\
2 & 3718.6 & 873.81 \\
3 & 2704.2 & 36.34 \\
4 & 566.1 & 0.5559 \\
5 & 33.585 & - \\
6 & 1 & - \\
\hline
\end{tabular}

1. Baud rate adopted in GE over copper. 


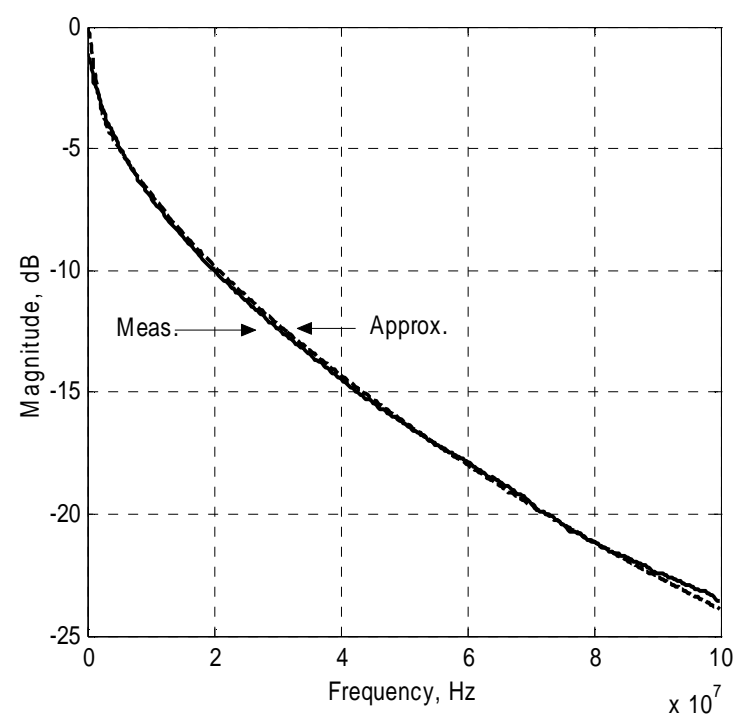

Fig. 4. Approximation of CAT5 cable frequency response.

\section{Modeling of Lumped Elements}

The modeling of channel lumped (discrete) elements; inductors, transformers, resistors and capacitors that appear in the line interface is considered next. Similar to the approach used in synthesis of active filters, the network is first split into a cascade of elementary two-ports consisting solely of series impedance $Z$, or shunt admittance $Y$. (To achieve this partitioning, all transformers in the circuit need to be replaced with their ladder equivalent). Each elementary two-port is linearly transformed using matrices (7), where $\beta$ is assigned a real impedance value, typically chosen from practical considerations. For convenience $\beta=R_{0}$ is employed here, with $R_{0}$ corresponding to the nominal characteristic impedance of the transmission line.

The elementary two-port LTE transfer functions can be obtained form (8), taking in account that the modified chain matrix of any series or shunt arm is of the following form

$$
T_{i}=\left[\begin{array}{cc}
1 & B_{i} \\
C_{i} & -1
\end{array}\right]
$$

where $B_{i}=-Z_{i}, C_{i}=0$ for any series arm $Z_{i}$, and $B_{i}=0, C_{i}=Y_{i}$ for any shunt arm $Y_{i}, \forall i$.

As all discrete elements are transformed using the same $\beta$, no port-matching is needed between the corresponding adjacent LTE modules, so simple cross-connect as shown in Fig. 3 is employed.

We will now consider the termination requirements for the first and last LTE blocks. A prototype signal source $E$ with resistance $R_{0}$ is transformed into a direct connection between the source and LTE input: $x_{11}=E$. If no source is active at the far end, output $y_{11} / 2$ would represent the chan- nel reflections, or echo. With the last two-port $N_{\mathrm{n}}$ terminated into a resistor $R_{0}$, the corresponding LTE output $y_{2 n} / 2$ with $x_{2 n}=0$ (grounded) would be equal the load voltage in the prototype. An open, or short circuit at the output of the last two-port $N_{\mathrm{n}}$ in the prototype would result in the corresponding LTE in full signal positive $x_{2 n}=y_{2 n}$, or negative $x_{2 n}=-y_{2 n}$ reflections respectively.

After all elementary two-ports and transmission line sections in the channel are transformed, the overall LTE model is obtained by interconnection of the adjacent LTE modules based on (9) or (16) depending on whether or not a discontinuity is present at the port boundary. In case of the discontinuity a port-matching module $J$ would be required between the adjacent LTE modules, otherwise a simple cross-connect shown in Fig. 3 is used. To illustrate the proposed method an application example is considered in the next section.

\section{Application Example}

An application example is given in this section whereby GE timing recovery test channel [1, p. 69] is modeled together with a typical transformer line interface. The channel consists of the relatively long $(100 \mathrm{~m}) 100 \Omega$ main UTP section appended at each end by a short "jumper" section with a characteristic impedance of $120 \Omega$. The discontinuities were purposely introduced in [1] such that the transceivers' loop-timed synchronization system could be tested under conditions of poor signal to echo ratio.

The test channel simplified circuit diagram (referred to as the prototype) is shown in Fig. 5(a). An exemplary transformer line interface is considered at each end of the channel with some parasitics: the transformer leakage inductance $L_{2,8}=200 \mathrm{nH}$ and a shunt capacitance $C_{3,7}=5 \mathrm{pF}$. Four discontinuity boundaries are introduced in the channel by the two short line segments with $120 \Omega$ characteristic impedance.

The prototype is first split into 9 sections, as indicated in Fig. 5(a) by the dashed lines, comprising of 3 homogeneous transmission line segments and 6 elementary two-ports, representing discrete instances. The associated port impedance values that are used in the respective transformation matrices (7) are shown on the top, whereas the line segment length figures are given at the bottom. Corresponding LTE transfer functions derived form (8) are presented in Fig. 5(b) below each prototype section. Whenever applicable, the transfer coefficients of port-matching blocks are also given.

Relatively short segments of line $T L_{4}$ and $T L_{6}$ have been assumed lossless and, hence, are modeled in the corresponding LTE as a pure delay with $\tau_{4}=0.76 \mathrm{UI}$ and $\tau_{6}=0.94 \mathrm{UI}$, where $\mathrm{UI}=8 \mathrm{~ns}$ corresponds to the Baud unit interval adopted in GE system. The main $100 \mathrm{~m}$ line segment introduces a delay of $\tau_{5}=70 \mathrm{UI}$ and is assumed here to be homogeneous for simplicity. 
(a)

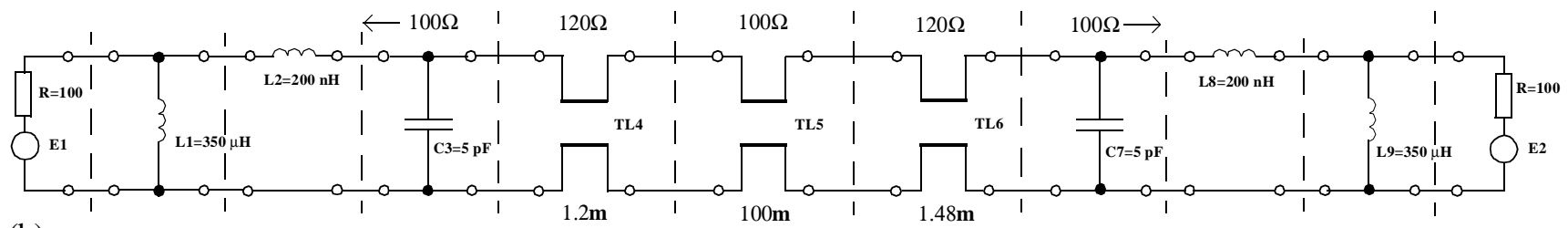

(b)

\begin{tabular}{|c|c|c|c|c|c|c|c|c|c|c|c|c|c|c|c|}
\hline LTE No & 1 & & 2 & 3 & J1 & 4 & J2 & 5 & $\mathbf{J 3}$ & 6 & J4 & 7 & 8 & & \\
\hline 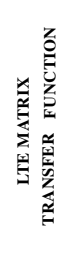 & 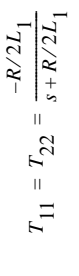 & 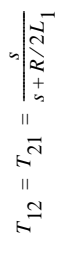 & 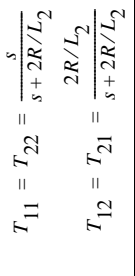 & 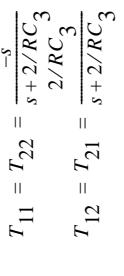 & 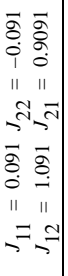 & 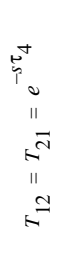 & 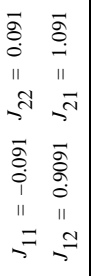 & 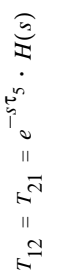 & 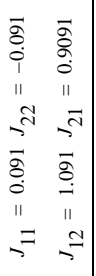 & 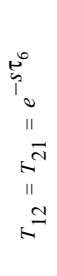 & 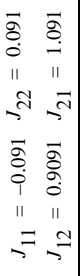 & 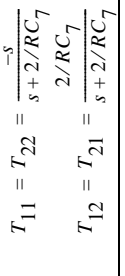 & 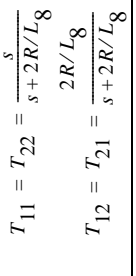 & 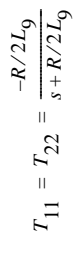 & 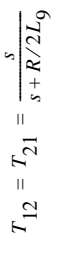 \\
\hline
\end{tabular}

(c)

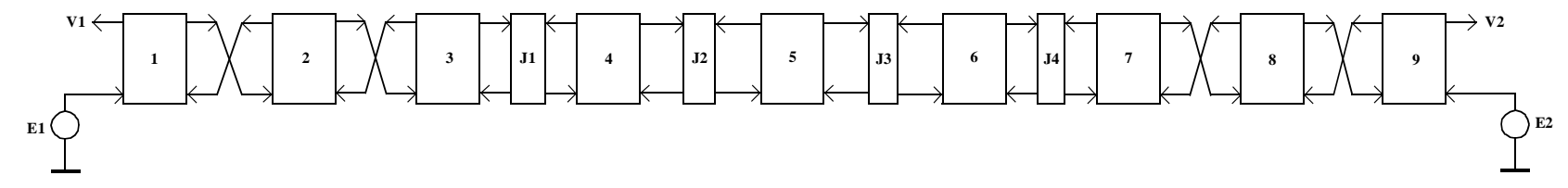

Fig. 5. (a) The GE timing recovery test channel circuit diagram—-the prototype. (b) The two-port corresponding LTE transfer functions. (c) Overall LTE model of the GE timing recovery test channel.

Recent studies indicate [10] that discontinuities due to the structural disorder in relatively long lines should be taken into account at high operating frequencies. This can be accomplished by further subdivision of large transmission line segments into a relatively small sections, which can be assumed homogeneous within their respective length.

The overall LTE model with properly connected LTE and port-matching blocks is shown in Fig. 5(c). Just like its twowire prototype the model is bi-directional in nature. This allows for full duplex transmission simulation in the channel when the sources $E_{1}$ and $E_{2}$ are both activated. In this case the model outputs $V_{1}$ and $V_{2}$ would represent a superposition of the echo and receive signals.

It should be noted that component value variations in the prototype would result in the transfer function coefficient variations in the corresponding LTE. As shown in [11] the LTE model sensitivity to the coefficient variations can be made exactly the same as the respective component sensitivity in the prototype, subject to LTE realization conditions. This important property allows realistic what-if analysis in the LTE model. The diagram in Fig. 5(c) has been realized using sensitivity adequate building blocks shown in Fig. 6
(LTE No. 1, 3, 7, 9) and Fig. 7 (LTE No. 2, 8).

The model in Fig. 5(c) was simulated in Spice and Matlab/ Simulink environment. For both Spice and Simulink the simulation results were identical within the computational precision. Fig. 8 shows simulated test channel echo impulse response compared to the properly terminated homogeneous line channel: reflection wave due to characteristic impedance discontinuity can be clearly observed on the graph. Corresponding frequency responses are shown in Fig. 9. A degradation of approximately $10 \mathrm{~dB}$ in echo frequency response in the test vs homogeneous channel can also be observed around $20 \mathrm{MHz}$ frequency range.

\section{Conclusions}

A method based on linear transformations of network port variables originally introduced for synthesis of active filters has been augmented and applied to behavioral modeling of transmission line channels with distributed and lumped discontinuities. Generic simulation tools like Simulink or Spice can be used based on their standard library primitives to mo- 


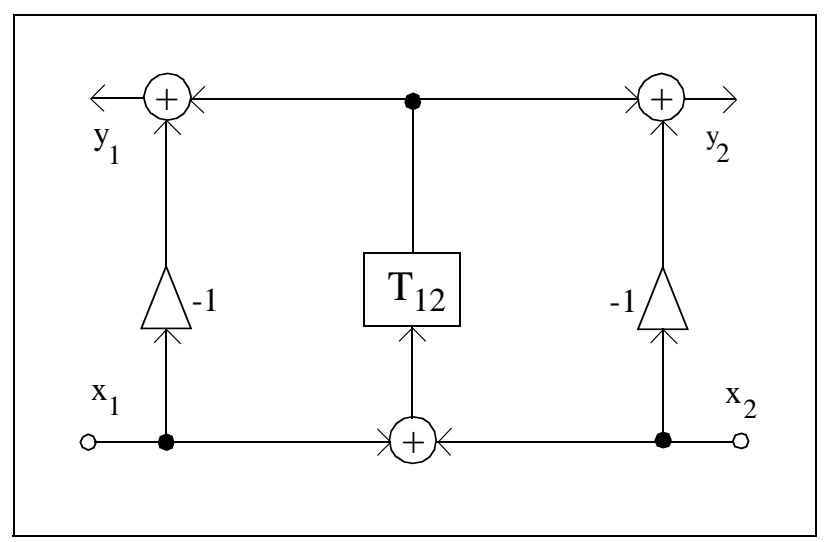

Fig. 6. LTE realization diagram for blocks No. 1, 3, 7, 9.

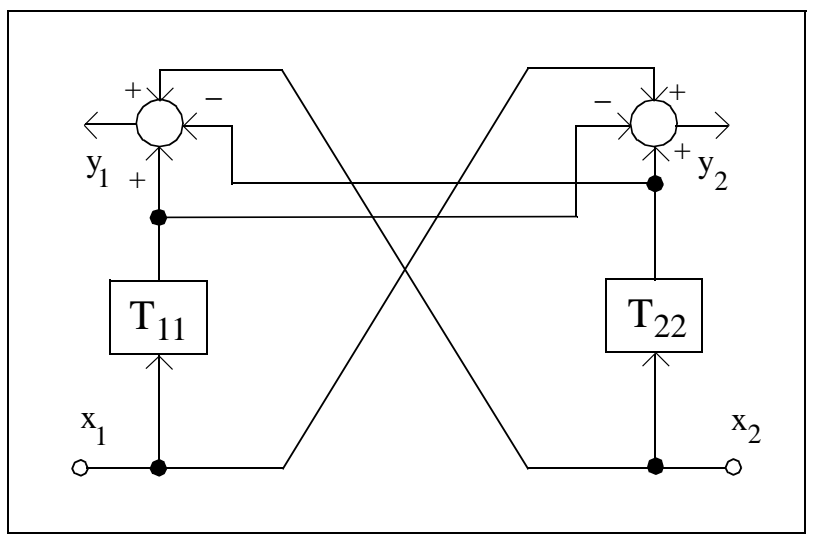

Fig. 7. LTE realization diagram for blocks No. 2, 8.

del physical phenomena in transmission lines such as skin effect, dielectric loss and characteristic impedance variations along with discrete basic circuit elements. Resulting continuous-time models provide for simulation of high speed data channels in mixed-signal systems, especially under conditions of sampling frequency drift and/or offset. Some additional work is need to extend the method for modeling of transmission line channels with bridged taps.

\section{References}

[1] Physical Layer Parameters and Specifications for $1000 \mathrm{Mb} / \mathrm{s}$ Operation Over 4-Pair of Category 5 Balanced Copper Cabling, Type 1000BASE-T, IEEE Std 802.3ab, 1999.

[2] Transmission and Multiplexing (TM); Access Transmission on Metallic Access Cables; Very high speed Digital Subscriber Line (VDSL), ETSI Standard TS 101 270-2, 2001, http://www.etsi.org.

[3] H. G. Dimopoulos and A. G. Constantinides, "Linear Transformation Active Filters," IEEE Trans. Circuits Syst., vol. CAS-25, pp. 845-852, 1978.

[4] W. Y. Chen, J. L. Dixon, D. L. Waring, "High Bit Rate Digital Subscriber Line Echo Cancellation," IEEE J. Select. Areas Commun., vol. 9, no. 6, pp. 848-860, 1991.

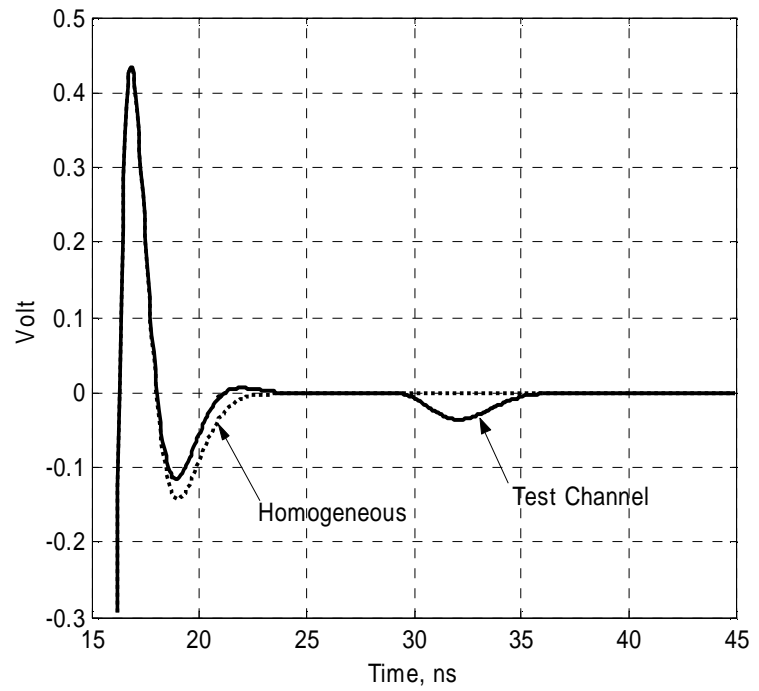

Fig. 8. Test vs homogeneous channel echo impulse response.

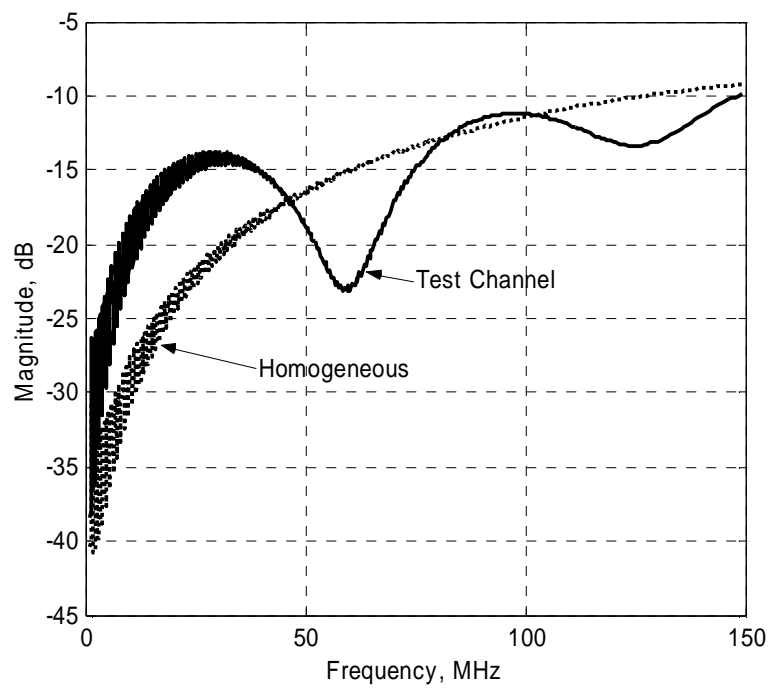

Fig. 9. Test vs homogeneous channel echo frequency response.

[5] J. A. C. Bingham, ADSL, VDSL, and Multicarrier Modulation, New York, Wiley, 2000.

[6] H. Wupper and K. Meerkötter, "New Active Filter Synthesis Based on Scattering Parameters," IEEE Trans. Circuits Syst., vol.CAS-22, pp. 594-602, 1975.

[7] N. S. Nahman and D. R. Holt, "Transient Analysis of Coaxial Cables Using the Skin Effect Approximation $A+B \sqrt{s}$, IEEE Trans. on $C T$, vol. 19, pp. 443-451, 1972.

[8] Q. Yu, O. W. Wing, "Computational Models of Transmission Lines with Skin Effects and Dielectric Loss," IEEE Trans. Circuits Syst., I, vol. 41, pp. 107-119, 1994

[9] Signal Processing Toolbox User's Guide, The MathWorks, 1996.

[10]F. Wenger, T. Gustafsson, L. J. Svensson, "Perturbation Theory for Inhomogeneous Transmission Lines," IEEE Trans. Circuits Syst., I, vol. 49, pp. 289-297, 2002

[11] A. Vareljian, "Development of Synthesis Methods for the High Order Active RC-filters," (in Russian), Ph.D. dissertation, "MEI", Moscow, 1989 